\title{
MODELO PARA A RECOMENDAÇÃO DE FERTILIZANTES NA CULTURA DA BATATA: MÓDULO REQUERIMENTO
}

\section{Diana Urrea Ramírez ${ }^{1} \bigotimes$, Victor Hugo Alvarez V¹, Júlio Cesar Lima Neves¹}

1 Universidade Federal de Viçosa, 36.570-000 -Viçosa - MG (Brasil)

$\triangle$ dpurrear@gmail.com

\section{PALAVRAS-CHAVE} modelagem, empíricomecanístico, FerticalcBatata, Solanum tuberosur

\section{RESUMEN}

O FERTICALC-Batata foi desenvolvido, para obter um modelo matemático que seja mais concordante com a realidade. O objetivo do trabalho foi calcular o módulo requerimento para a cultura da batata por meio da recopilação de informações disponíveis na literatura científica. A demanda da planta por um nutriente i (dNui) resulta do somatório dos conteúdos (c) dos nutrientes (i) nos diferentes compartimentos da planta (j) (tubérculo (t), folhas (f), caules (c) e raízes ( $r$ ) com o que posteriormente será calculada a demanda total da planta. Foi calculada a demanda para Solanum tuberosum subsp. tuberosum, para 8 estratos de produtividades que são 15, 20, 25, 30, 35, 40, 45 e 50 t/ha, para os macronutrientes e os micronutrients os CUB (coeficientes de utilização biologica) encontrados por planta espresados em (kg/kg) são: $N(163,29 ; 159,59), P(1793,91 ; 1207,80), K(102,51 ; 117,97)$, Ca (1622,72; 823,62), Mg $(1982,73 ; 1272,38) S(2448,29 ; 1732,96)$, os resultados confirmam a alta demanda da cultura pelo K que dentre os dados é o CUB mais baixo, indicando que por $\mathrm{kg}$ de nutriente utilizado produz a menor quantidade de massa de matéria seca, esses coeficientes se consideram altos ao ser comparados com os obtidos para outras culturas como melão que para $N, P, K, \mathrm{Ca}, \mathrm{Mg}$ e S foram 66, 189, 30, 96, 485 e $297 \mathrm{~kg} / \mathrm{kg}$ respectivamente, indicando a maior eficiencia da batata na utilização dos nutrientes. Com 0 uso destes coeficientes será estimada a demanda total da planta com as taxas de recuperação obtidas também da literatura.

\section{MODELO DE RECOMENDACIÓN DE FERTILIZANTES EN LA CULTIVO DE PATATA: MÓDULO DE REQUERIMIENTOS.}

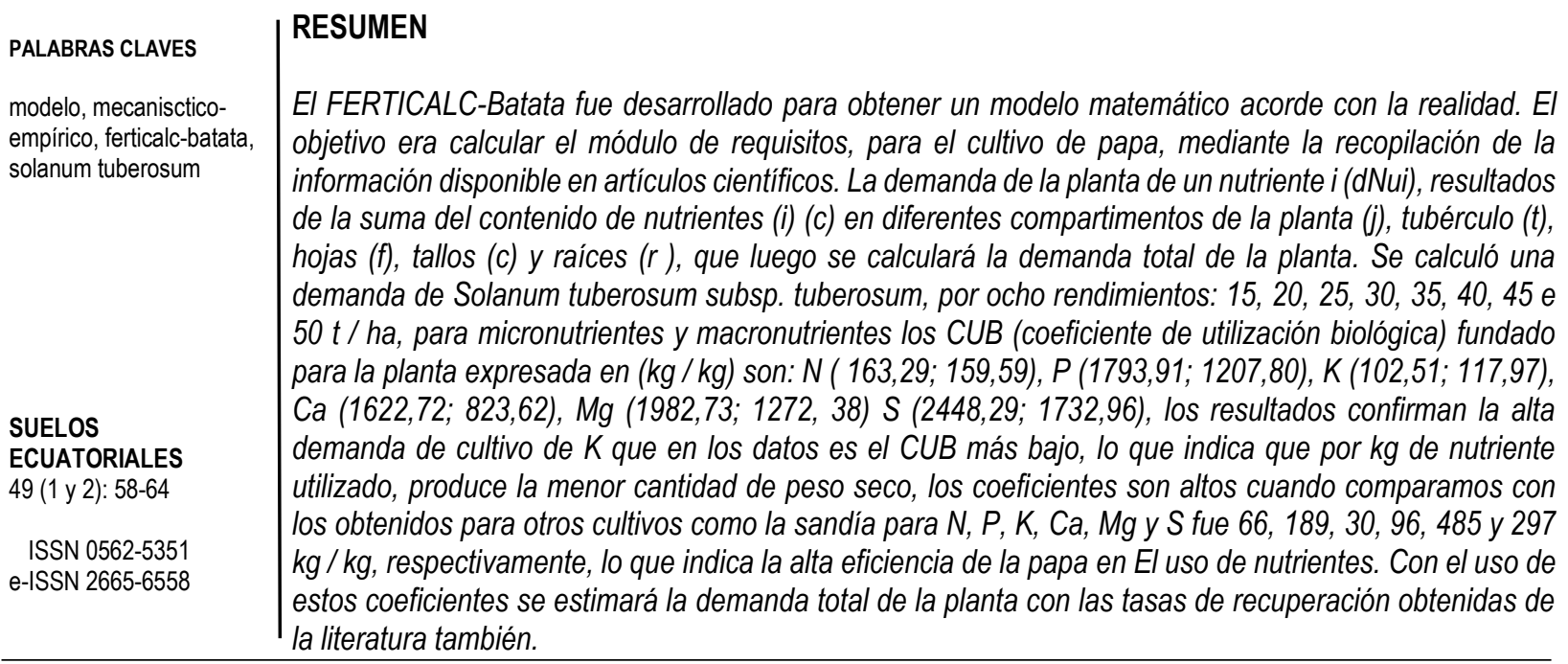




\section{INTRODUÇÃO}

No Brasil, a cultura da batata foi introduzida por imigrantes europeus no final do século XIX, no sul do país (Pereira, 2011).

Apesar de, a batata ser considerada apenas mais um item do grupo das hortaliças no Brasil, mundialmente, sua importância em termos de consumo humano é comparável à de grandes culturas, ficando atrás somente do trigo, do arroz e do milho (Quadros, 2007). É um dos dez alimentos mais importantes produzidos nos países em desenvolvimento (Espinal et al., 2005), cultivada em cerca de 130 países e consumida por mais de um bilhão de pessoas (CIP, 2011).

A adubação da cultura da batata é uma prática agronómica importante, permite incrementar notavelmente 0 rendimento e a qualidade dos tubérculos coletados, precisando de uma inversão importante em capital (Sierra et al., 2002). Segunda a ABBA (2008), a bataticultura brasileira não adota critérios técnico-científicos quando se fala em correção do solo e nutrição mineral da planta. Esse fato pode comprometer a produtividade e afetar significativamente o custo de produção, pois, 0 consumo relativo de fertilizantes por unidade de área na cultura é impressionante, ocupando o primeiro lugar no "ranking" dentre as principais culturas, pelo que se deve dar especial atenção a este aspecto.

$\mathrm{Na}$ prática, as formas de recomendação da adubação estão baseadas no uso de tabelas com

\section{MATERIAIS E MÉTODOS}

\section{Fundamento do FERTICALC}

Utilizando os princípios do balanço nutricional, 0 FERTICALC-Batata combina modelos mecanísticos e empíricos, para alimentar o sistema, utilizando a menor quantidade de informações possíveis, sem comprometer a exatidão das recomendações (Lima, 2012).

As recomendações a partir do FERTICALC-Batata estão baseadas no balanço nutricional entre perdas e ganhos de nutrientes no sistema solo-planta, que será obtido pela diferença entre o requerimento do calibrações regionais de acordo com características do solo, como textura, ou da disponibilidade de nutrientes encontrados nas analises, e também com - cultivar. 0 método procura fazer uma generalização de acordo com classes de fertilidade, resultando em inconvenientes derivados das características diferenciais entre umas e outras localidades, que podem estar numa mesma classe. Além do fato que a necessidade de fertilização varia de acordo com a produtividade esperada, assim, é necessário ter um modelo que seja mais concordante com a realidade da dinâmica do solo, a interação com a planta e o esgotamento dos nutrientes.

Atualmente, de acordo a ideia proposta por Barros et al., (1995), com o software NUTRICALC para a cultura de eucalipto, e posteriormente adotada por Novais \& Smyth (1999), para o cálculo do requerimento de $P$, o departamento de solos da UFV desenvolveu o Sistema de Recomendação de Corretivos e Fertilizantes FERTICALC, que procura resolver os problemas derivados do uso de tabelas, considerando assim, a dinâmica do solo, o seja o suprimento de nutrientes na fração mineral e orgânica, e o requerimento da cultura de acordo com a produção de matéria seca total por quilograma de nutriente absorvido e utilizado.

Diante do exposto, o objetivo do trabalho foi aprimorar o sistema de recomendação de corretivos e fertilizantes para a cultura da batata.

nutriente pela cultura e seu suprimento pelo solo, os resíduos vegetais e a matéria orgânica.

0 FERTICALC-Batata expressa tanto 0 requerimento (rq), quanto o suprimento para cada nutriente (su) em termos de dose, devendo ser a diferença dentre ambos iguais à dose recomendada do nutriente (drNui) via fertilizante (F. Oliveira, et al., 2003).

O requerimento (rqNui) é a demanda (dNui) dos nutrientes corrigidas pela taxa de recuperação dos mesmos pela planta (trNui_PI) (Alvarez V. et al., no prelo), para atingir uma produtividade determinada, por ciclo de produção (Equação 1). 
rqNui = dNui / trNui_PI

$$
\text { (Equação 1) }
$$

A dNui resulta do somatório dos conteúdos (c) dos nutrientes (i) nos diferentes compartimentos da planta (j), para o caso da batata os compartimentos considerados foram: tubérculo $(t)$, folhas $(f)$, hastes (h) e raíces (r), de modo que a demanda será obtida pela equação 2 , que se mostra a seguir: $\mathrm{dNui}=\mathrm{cNui} \_\mathrm{t}+\mathrm{cNui} f \mathrm{f}+\mathrm{cNui} \_\mathrm{h}+\mathrm{cNui} \_\mathrm{r}$ (Equação 2)

O conteúdo é o resultado de dividir a produtividade esperada no compartimento (j) pelo coeficiente de utilização biológica (CUB) do nutriente (i): cNuij = Produtividade esperadaj $/$ CUBij (Equação 3)

E a trNui_PI, do nutriente aplicado ao solo como fertilizante, é considerada, pois a planta não absorve $100 \%$ do que foi disponibilizado devido a fatores como perdas e competição do solo com a planta (F.C. Santos et al., 2008), estas taxas são uma medida da eficiência da planta, o valor por nutriente será tomado de trabalhos de pesquisa.

\section{RESULTADOS}

\section{Requerimento do nutriente pela planta}

Requerimento do nutriente pela planta

O sistema FERTICALC-Batata estimou 0 requerimento rqNui para a cultura apresentado no quadro 1. No entanto, os requerimentos não são fixos, pois são o resultado de dividir a demanda pela trNui_PI, sendo que estas taxas são igualmente dependentes do valor de Fósforo remanescente $(P$, $\mathrm{S}$ e $\mathrm{Zn}$ ) que é uma medida do
Por outro lado, o módulo suprimento considera três tipos de fontes de nutrientes para a cultura; 0 suprimento pelas formas disponíveis no solo (suNui_Sol), pela matéria orgânica (suNui_MOS) e pelos resíduos vegetais (suNui_Res), resultando na equação 4:

suNui = suNui_Res + suNui_MOS + suNui_Sol

(Equação 4)

O suNui_Sol se obtem com o teor $(t)$ do nutriente encontrado nas analises, e corrigido pela taxa de recuperação do extrator (trNui_Ext)

0 balanço no FERTICALC-Batata pode ser sumarizado assim:

drNui= (rqCul + rqSus) $-($ suDisp + suMO + suROrg) (Equação 5)

Em que: rqCul : Requerimento da cultura; rqSus: Requerimento de sustentabilidade; suDisp: Suprimento pelo disponível; suMO: Suprimento pela matéria Orgânica; suROrg: Suprimento pelos Resíduos Orgânicos poder tampão do solo, ou da produtividade ( $\mathrm{N}, \mathrm{K}$, $\mathrm{Ca}, \mathrm{Mg}, \mathrm{B}$ ) e em outros casos são fixas (Fe, Mn e $\mathrm{Cu}$ ), para obtê-las, as equações foram tomadas e adaptadas dos outros FERTICALC.

Já que o rqNui é variável em função da trNui_PI, foram feitas figuras para representar a demanda $\mathrm{e}$ não o requerimento da batata (Figura 1), já que a demanda é fixa para cada uma das produtividades consideradas. 
Quadro 1. Requerimento total de nutrientes pela planta, utilizados no FERTICALC-Batata, utilizando trNui_PI, calculadas com P-rem de $4 \mathrm{mg} / \mathrm{L}$

\begin{tabular}{ccccccccccccc}
\hline \multicolumn{10}{c}{ REQUERIMENTO } \\
\hline Prod. \\
(t/ha)
\end{tabular}

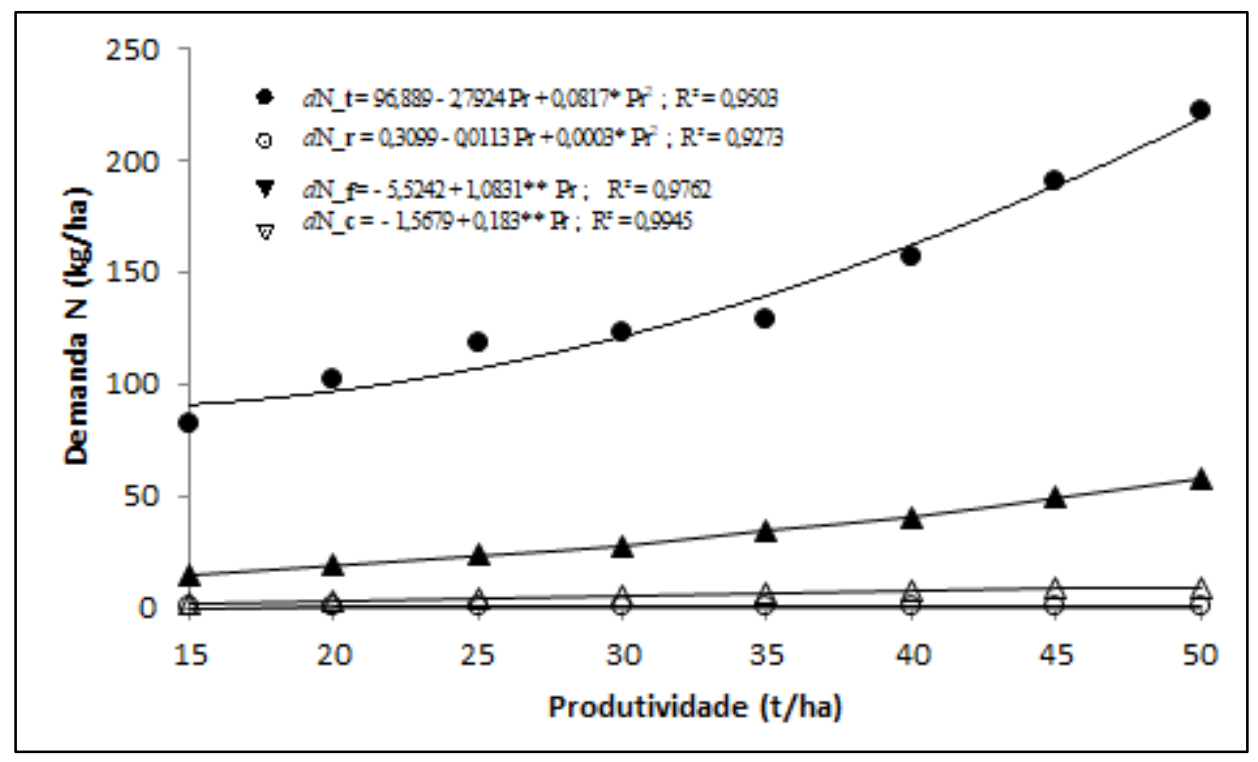



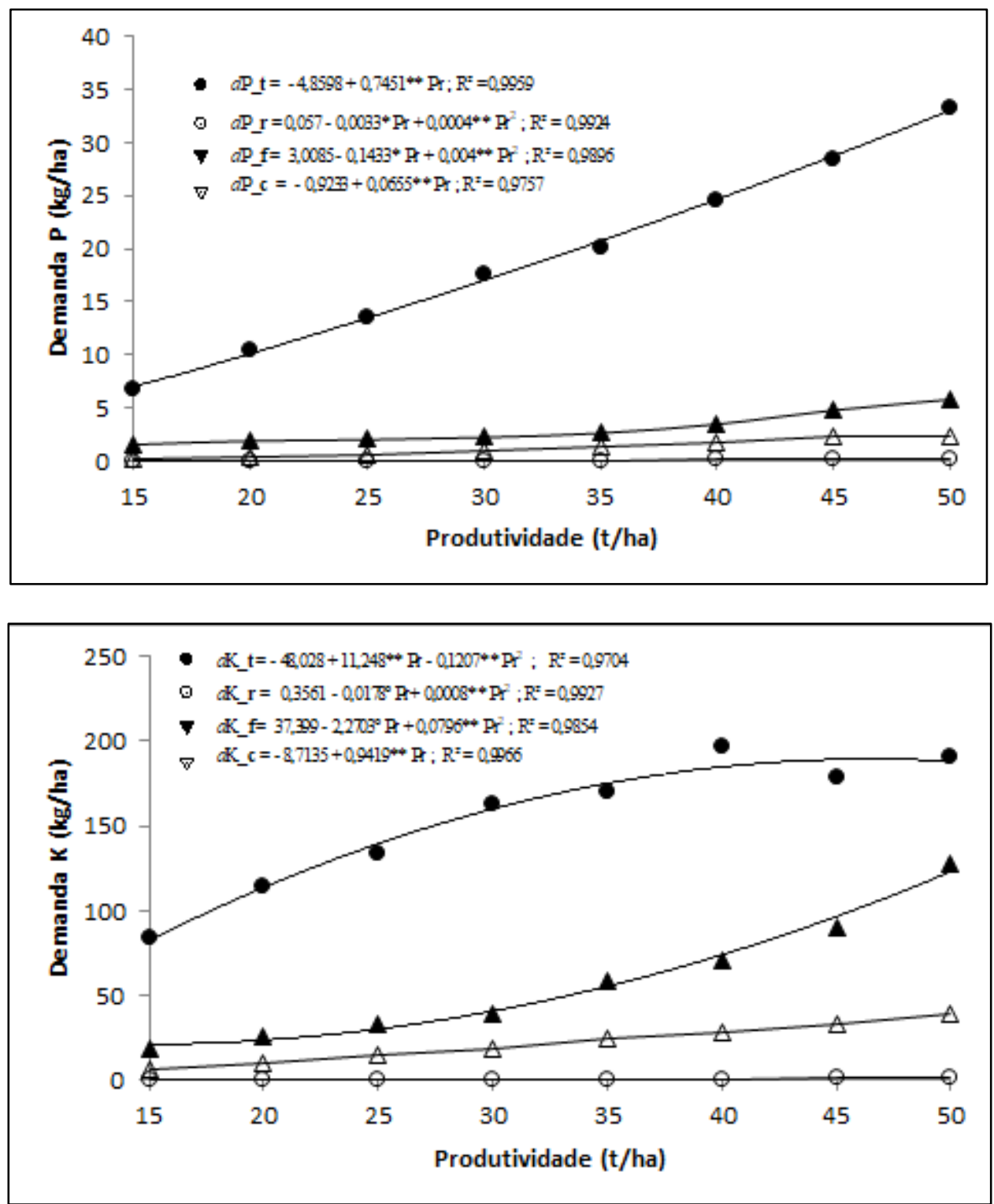

Figura 1. Demanda de N, P e K, em função da produtividade em t/ha, para cada um dos compartimentos da batata, tubérculo (t), raíz (r), folha (f) e caule (c).

De acordo com os valores obtidos com o uso da equação para estimar a trP_Pl, a eficiência aproximada da cultura para o P é de 9 até $30 \%$ dependendo do P-rem (4 e $50 \mathrm{mg} / \mathrm{L})$. Segundo Dechassa et al (2003), a batata tem sido considerada com escassa habilidade de absorver $P$ em solos com pouca disponibilidade, possivelmente pela baixa densidade de raízes, as quais se desenvolvem principalmente nos primeiros $30 \mathrm{~cm}$ de profundidade, até agora os dados de recuperação obtidos em pesquisas específicas para a cultura vão de $3-4,5 \%$, em solos com alta disponibilidade de $P$, que foram dependentes da cultivar utilizada, tanto que a adição de doses muito elevadas (> $500 \mathrm{~kg} / \mathrm{ha}$ P2O5) diminuiu a eficiência da planta na utilização do nutriente (Fernandes, 2013). 
De outro lado, Castro (2005), encontrou que dependendo da fonte de adubo utilizado, a eficiência na recuperação do $\mathrm{N}$ pela planta, variou desde 30 até $95 \%$, diferente do FERTICALC-Batata onde as trN_PI vão desde $73 \%$ até $81 \%$, dependendo da produtividade desejada, no entanto essas eficiências estão sujeitas a uma série de fatores que não estão considerados para esta versão do FERTICALC como a época de aplicação, lixiviação após irrigação e chuva, parcelamento da adubação, fontes utilizadas, entre outros fatores que interferem com a eficiência da planta em converter o nutriente aplicado em matéria seca vegetal.

Para os outros nutrientes, ainda não há estudos, dos quais seja possível obter uma referencia sobre a eficiência da batata para absorver os nutrientes, pelo que foi feito o cálculo das trNui_Pl, acreditando-se, na efetividade que as equações utilizadas têm mostrado até agora para a estimativa do requerimento, nas outras pesquisas feitas no modelo FERTICALC.

Fazendo a comparação dos quatro compartimentos avaliados tanto para macro como para micronutrientes (Figuras 1), é evidente a superioridade do tubérculo como órgão extrator na planta de batata, também, que a tendência geral, é o incremento da demanda junto com o acréscimo da produtividade, situação esperada, pois com maior

\section{CONCLUSÕES}

0 desenvolvimento do módulo requerimento permitiu conhecer a demanda da planta pelos nutrientes, dentre eles o $\mathrm{K}$, que é requerido em maior quantidade, também que o requerimento está variando amplamente de acordo com a produtividade desejada. produção a planta precisa de uma maior quantidade de nutrientes para gerar maior massa de matéria seca.

Para o tubérculo, se for comparada a extração dentre os macronutrientes, $\mathrm{N}$ (de 65,25 até 165,03 $\mathrm{kg} / \mathrm{ha}$; menor e maior produtividade avaliadas), $\mathrm{P}$ (de 6,73 até $33,35 \mathrm{~kg} / \mathrm{ha}$ ), $\mathrm{K}$ (de 83,90 até 190,21 kg/ha), $\mathrm{Ca}$ (de 2,35 até $13,57 \mathrm{~kg} / \mathrm{ha}$ ), $\mathrm{Mg}$ (de 3,17 até 14,72 $\mathrm{kg} / \mathrm{ha}$ ) e $\mathrm{S}$ (de 4,07 até 12,54 kg/ha), é obvia a maior demanda pelo $\mathrm{K}$, o que resulta lógico, analisando a composição média da $\mathrm{mMs}$ do tubérculo, em que 0 $\mathrm{K}$ chega até $56 \mathrm{~g} / \mathrm{kg}$, comparado com $\mathrm{P}$ e Ca que representam 0,5 g/kg e 0,09 $\mathrm{g} / \mathrm{kg}$ respectivamente (ABBA, 2001).

No FERTICALC os índices da colheita foram de até $85 \%$, dependendo do elemento analisado, sendo $\mathrm{N}$ (IC $=82$ até $74 \%), P(78$ até $80 \%$ ) e K (de 77 até $53 \%)$, os nutrientes que finalmente são transloucados em maior quantidade até o tubérculo, como é esperado, pela importância destes elementos nas funções metabólicas da planta. A partição dos nutrientes indica a importância de contabilizar na demanda, todos os compartimentos da cultura, visto que raízes, folhas e caules, podem representar até $26 \%$ da demanda total, com o que ao desconsiderá-los, está se subestimando 0 requerimento total da cultura.

O FERTICALC dá uma ideia da importância de contabilizar todos os compartimentos da planta no momento de fazer o desenvolvimento da dose recomendada, pois se for feita com a necessidade do tubérculo só, estaria se subestimando 0 requerimento da planta pelos nutrientes. 


\section{REFERENCIAS}

ALVAREZ, V., V.H., SANTOS, A.F., SANTOS, G.L. \& MATTA. P.M. (No prelo). Fertilização de Plantas Ornamentais: Método Requerimento Suprimento. R. Bras. Ci. Solo.

BARROS, N.F.; NOVAIS, R.F.; TEIXEIRA, J.L. \& FERNANDES, E.I. (1995). NUTRICALC 2.0 Sistema para cálculo del balance nutricional y recomendación de fertilizantes para el cultivo de eucalipto. Bosque, 16 (1): 129-131.

CASTRO, H. (2005). Balance y prospectiva de la investigación en la fertilización de papa en Colombia. Memorias "I Taller Nacional sobre suelos, fisiología y nutrición vegetal en el cultivo de la papa. Centro Virtual de Investigación de la Cadena Agroalimentaria de la Papa CEVIPAPA. pp $31-43$.

CIP, CENTRO INTERNACIONAL DE LA PAPA. (2011). 50 Potato Facts. 2011. Disponível em: http://www.cipotato.org/publications/pdf/004495.pdf . Acesso: 25 de janeiro de 2014.

DECHASSA, N.; SCHENK, M. K.; CLAASSEN, N. \& STEINGROBE, B. (2005). Phosphorus efficiency of cabbage (Brassica oleraceae L. var. capitata), carrot (Daucus carota L.), and potato (Solanum tuberosum L.). Plant and Soil 250: 215-224, 2003.

ESPINAL, C.; MARTíNEZ, H.; PINZÓN, N. \& BARRIOS, C. La cadena de la papa en Colombia. Ministerio de Agricultura y desarrollo rural. Bogotá, Colombia. 123 p.

FERNANDES, A.M. (2014). Crescimento, produtividade, acúmulo e exportação de nutrientes em cultivares de batata (Solanum tuberosum L.). 2010. 158 p. Universidade Estadual Paulista "Júlio de Mesquita Filho", UNESP, Botucatu, SP. Tese (Mestrado em Agronomia)
.FOOD AND AGRICULTURE ORGANIZATION OF THE UNITED NATIONS - FAO. FAO Statistical Databases. Disponível em http://faostat.fao.org/site 1567/ DesktopDefault.aspx?PagelD=567\#ancor. Acesso: 25 de janeiro de 2014

LIMA, J.A. (2012). Sistema de recomendação de adubos e fertilizantes para o meloeiro com base no balanço nutricional. Tese (Mestrado em Agronomia). Universidade Federal do Ceará; Ceará. $123 p$.

OLIVEIRA, F. H. T.; NOVAIS, R. F.; ALVAREZ V., V. H. \& CANTARUTTI, R. B. (2003). Desenvolvimento de um sistema para recomendação de adubação para a cultura da bananeira. Revista Brasileira de Ciência do Solo. Viçosa, v. 29, No. 1:131-143.

PEREIRA, A.S. (2011). A evolução da batata no Brasil. In: CONGRESSO BRASILEIRO DE OLERICULTURA, 51. Horticultura Brasileira 29. Viçosa: ABH.S5701-S5710.

QUADROS, D. A. (2007). Qualidade da Batata, Solanum tuberosum L., cultivada sob diferentes doses e fontes de potássio e armazenada em temperatura ambiente. Dissertação (Mestrado em Tecnologia de alimentos) - Universidade Federal do Paraná, Curitiba.

SANTOS F.C.; NEVES, J.C.L.; NOVAIS, R.F.; ALVAREZ, V., V.H. \& SEDIYAMA, C.S. (2008). Modelagem da recomendação de corretivos e fertilizantes para a cultura da soja. Revista Brasileira da Ciência do Solo, 32:1661-1674.

SIERRA, C.; SANTOS, J. \& KALAZICH, J. (2002). Manual Fertilización del cultivo de la papa en la zona sur de Chile. Instituto de investigaciones agropecuarias. Boletin INIA No. 76. 